\title{
Spinal Epidural Arteriovenous Fistula with Double Perimedullary Reflux
}

\author{
Jai Jai Shiva Shankar, Karel terBrugge, Timo Krings
}

Can J Neurol Sci. 2012; 39: 239-241

The most common vascular malformation of the spine is spinal dural arteriovenous fistulae which account for around $70 \%$ of vascular malformation of the spine. These have been recently classified as "lateral epidural" shunts given their location along the lateral nerve root sleeves ${ }^{1}$. According to this classification, the ventral epidural AV shunts that drain into the ventrally located epidural venous plexus are usually asymptomatic, which has been previously attributed to the presence of an anti-reflux mechanism within the dural sleeves that prevents retrograde flow of the arterialized blood into radicular or perimedullary veins ${ }^{2}$.

There are a few case reports in the literature that describe the rare occurrence of a shunt of the ventral spinal epidural space being redirected via a radicular vein towards the perimedullary veins resulting in venous congestion of the $\operatorname{cord}^{3}$. In the reported cases, a single radicular vein was rerouting the blood from the epidural plexus towards the spinal cord.

Herewith, we report a patient with an epidural arteriovenous shunt with retrograde drainage into perimedullary veins via two radicular veins at two different levels, leading to the classic clinical and magnetic resonance imaging (MRI) findings of venous congestion of the spinal cord. To the best of our knowledge, this is the first description of two separate radicular veins being present to reroute the blood from the epidural venous plexus towards the spine.

\section{Case Report}

A 68-year-old gentleman presented with a two month history of progressive paraparesis and paraesthesia. At the time of admission he was unable to walk being wheel chair bound since two weeks before admission. He had lost control of sphincter functions two weeks prior to admission. No further deficits were detected. His past history was significant with diagnosis of hepatocellular carcinoma one year prior to this event for which he declined any treatment.

On MRI, the thoraco-lumbar spinal cord was slightly swollen with T2- hyperintensity and T1 hypo- to iso-intensity (Figure a). Post contrast T1 weighted images showed pronounced diffuse enhancement of the lower thoraco-lumbar spinal cord with the enhancement being more pronounced in the lower part of the cord including the conus medullaris (Figure b). Serpentine perimedullary hypointensities were present on T2-weighted studies which showed enhancement on contrast-enhanced T1weighted images, indicating perimedullary dilated veins posterior and anterior to the spinal cord (Figure a, b). No intramedullary nidus was detected. ATECO MR angiogram was non-conclusive in localizing the level of the fistula. However a large venous pouch, perimedullary dilated veins and a suspicious prominent segmental artery was seen on the multi-phase computed tomography (CT) scan of the abdomen performed for the assessment of his hepato-cellular carcinoma (Figure c).

Based on the information of the CT scan targeted spinal angiography was performed with injection of contrast agent into the intercostal arteries from T12 to L3 level bilaterally. An epidural shunt was seen on the right L1 and L2 segmental artery without an intervening nidus (Figure d, e). This shunt was supplied by the radiculomeningeal artery at the right L2 level and drained first into a large epidural venous pouch extending from L2 to L4 vertebral body level. The shunt was then draining into multiple epidural segmental veins at the L2 to L4 levels. Drainage of the epidural venous pouch into a radicular vein at the right L3 level was also seen which eventually connected to the perimedullary veins (Figure e). There was another small tortuous vein adjacent to the first radicular vein which appeared to be connecting the venous pouch with the perimedullary veins (Figure e).

Since the feeding artery was very small in size, the endovascular treatment was not contemplated. The patient underwent a partial L-3 laminectomy. After opening the dura mater medially, an arterialized intradural radicular vein was identified and followed to the fistulous point on the right side as shown on the angiogram. This was coagulated and dissected. The epidural venous pouch was not coagulated as it could not be completely exposed at surgery. The second intradural radicular vein could not be visualized at surgery so left untreated.

Spinal angiography performed three days after surgery revealed complete closure of the primary perimedullary reflux (Figure f). However the second relatively smaller and more tortuous venous reflux continued to be present with opacification of the secondary (smaller) perimedullary venous system. Since this vein was not seen at the first surgery, a repeat surgery was offered to the patient. Given the underlying disease with uncertain long-term life expectancy and the fact that the patient did not change clinically after the first surgery, the family opted for clinical observation and no further treatment.

From the Department of Diagnostic Imaging (JJSS), Department of Medical Imaging (KtB, TK), Division of Neuroradiology, Toronto Western Hospital, Toronto, Ontario, Canada.

Received August 8, 2011. Final Revisions Submitted October 18, 2011. Correspondence to: Jai Jai Shiva Shankar, Department of Diagnostic Imaging, Division of Neuroradiology , QE II Hospital, 1796 Summer Street, Room No-3305A, Halifax, Nova Scotia, B3H 3A7, Canada. 

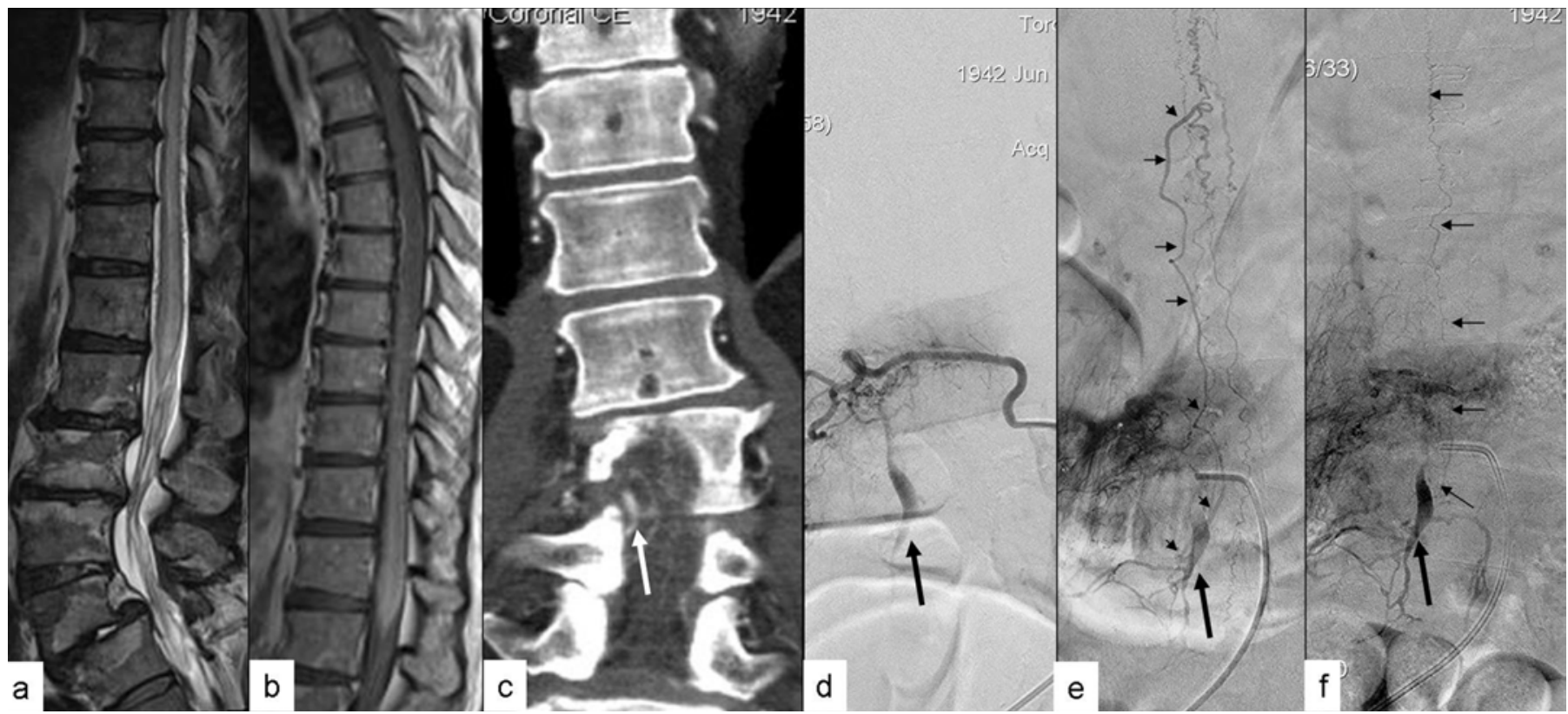

Figure: MRI of thoraco-lumbar spine showing (a) Slightly swollen with T2- hyperintensity in the cord with multiple flow voids in the subarachnoid space. (b) Post contrast T1 weighted images showed pronounced diffuse enhancement of the lower thoraco-lumbar spinal cord with enhancement on the surface of the spinal cord indicating perimedullary dilated veins. (c) Multi-phase computed tomographic (CT) scan of the abdomen performed for the assessment of his hepato-cellular carcinoma showing a large venous pouch (white arrow) and perimedullary dilated veins. (d,e) Right L2 segmental artery AP angiogram showing an epidural shunt draining first into a large epidural venous pouch extending from L2 to L4 vertebral body level (large arrow). The epidural venous pouch is draining into a radicular vein at the right L3 level was also seen which eventually connected to the perimedullary veins (small arrows). (f) Post operative check spinal angiography three days after surgery showing complete closure of the primary perimedullary reflux. However the second relatively smaller and more tortuous venous reflux from the epidural venous pouch (large arrow) continued to be present with opacification of the secondary (smaller) perimedullary venous system (small arrows).

\section{Discussion}

The epidural venous plexus continues as a single system from the skull base to the sacrum is located in the epidural fatty connective tissue. The perimedullary veins drain into the epidural venous plexus through multiple segmental (radicular) veins. These veins may or may not follow the nerve roots and thus there are emissary veins which may connect the perimedullary venous plexus with the epidural venous plexus at an intersegmental level. The epidural venous system is a valveless system and drains into the azygous and hemiazygos venous system by segmental veins. Normally the venous blood flows antegradely from the perimedullary venous system to the epidural venous system and then to the azygous venous system. The retrograde flow from the epidural venous plexus to the perimedullary veins is usually not observed and has only rarely been described in the literature. This unusual retrograde flow could reflect failure of the anti-reflux mechanism ${ }^{4}$.

The nature of this anti-reflux mechanism is not entirely understood. Tadie et $\mathrm{al}^{4}$ have excluded the presence of venous valves, and described an anti-reflux system within the transdural course of the radicular vein, resulting from inherent narrowing and a zigzag course of the vein while crossing the dura. This was subsequently demonstrated on micro-angiography and histological examination. The zigzag course results either from the proximity of a nerve root or from the presence of a bulge of dural collagenous fibers with a glomus-like appearance.
Therefore, differentiation into a slit- or bulge-type valve was made $^{2}$.

Since asymptomatic epidural shunts without reflux to the perimedullary veins may occasionally be found during spinal angiography, the anti-reflux mechanism normally works even in the presence of an arterialized epidural plexus. These epidural shunts continue to be asymptomatic if the anti-reflux mechanism remains intact.

In a review of the literature we found five case reports in which the findings were similar. Chen et $\mathrm{al}^{5}$ and Cognard et $\mathrm{al}^{6}$, each reported on a patient with a paraspinal AVF that caused hypertension of the intrathecal venous system, although in neither patient was cord edema present. In the patient described by Chen and coworkers, a paraspinal AVF that originated from the $\mathrm{T}-12$ intercostal artery drained into the external epidural plexus without an obvious reflux into perimedullary veins. They argued that the increased pressure within the epidural plexus might have been sufficient to cause symptoms (numbness of distal extremities). Cognard et $\mathrm{al}^{6}$, were able to show retrograde filling of intradural veins in a patient with a high-flow lesion. Whereas venous congestion was absent and paraspinal shunts were present in these two patients, the other two patients described in the literature had a spinal epidural AVF that showed secondary reflux into perimedullary veins and clinical signs of spinal venous hypertension ${ }^{2,7}$. In the third case, the lateral sacral artery shunted into the epidural plexus and a retrograde filling of 
the S-1 medullary veins was noted ${ }^{7}$. Surgery was needed to obliterate the venous reflux. The fourth case was a patient where an epidural arteriovenous shunt showed delayed retrograde drainage into perimedullary veins, leading to the classic clinical imaging findings of venous congestion ${ }^{2}$. This patient underwent surgical disconnection of both the epidural shunt zone and the radicular vein resulted in complete obliteration of the fistula. The fifth patient presented with acute paraplegia and had an exclusive epidural fistula with perimedullary venous drainage. This patient underwent trans-arterial embolization and resulted in complete recovery of the paraplegia ${ }^{3}$.

The cases described in the literature have shown evidence of perimedullary venous reflux through a single radicular vein. In our case there were two routes of the venous reflux from the epidural space towards the perimedullary veins. The possible reason for this could be the failure of anti-reflux mechanism due to atypical anatomical disposition in the adjacent segmental level due to some segmental vulnerability. The high venous pressure could also result in the failure of the anti-reflux mechanism since both of the veins were connected to the same epidural venous pouch. This rare phenomenon highlights the fact that following surgical disconnection of these lesions follow-up spinal angiography is still necessary to rule out the possibility of a persistent shunt which may still lead to venous congestion of the cord. It may also suggest that the way to successfully cure the fistula is to attempt endovascular treatment with glue (as opposed to the surgical disconnection of veins which leaves open the possibility that an additional arterialized venous vessel might be missed, as in this case), which is safe when there are no arterial supply to the cord from the same level.

We conclude that the AV shunting into an epidural plexus with subsequent reflux into intradural perimedullary veins is a rare finding that can only be diagnosed by meticulous selective spinal angiography and extensive imaging studies to demonstrate the delayed retrograde filling of the perimedullary vein that causes the symptoms. Since more than one vein can be used to reroute the arterialized blood from the epidural venous plexus to the perimedullary veins, follow-up spinal angiography is necessary to exclude persistent venous hypertension.

\section{REFERENCES}

1. Geibprasert S, Pereira V, Krings T, et al. Dural arteriovenous shunts: a new classification of craniospinal epidural venous anatomical bases and clinical correlations. Stroke. 2008; 39(10):2783-94.

2. Krings T, Mull M, Bostroem A, Otto J, Hans FJ, Thron A. Spinal epidural arteriovenous fistula with perimedullary drainage. Case report and pathomechanical considerations. J Neurosurg Spine. 2006; 5(4):353-8.

3. Reul J, Braun V. Spinal arteriovenous epidural fistula with acute paraplegia. Diagnosis and neurointerventional emergency treatment. A case report. Interv Neuroradiol. 2007; 13(1):75-8.

4. Tadie M, Hemet J, Freger P, Clavier E, Creissard P. Morphological and functional anatomy of spinal cord veins. J Neuroradiol. $1985 ; 12: 3-20$

5. Chen CJ, Huang CC, Hsu YY, Hsu WC. Small isolated para-spinal arteriovenous fistula. AJNR Am J Neuroradiol. 1997; 18:359-61.

6. Cognard C, Semaan H, Bakchine S, et al. Paraspinal arteriovenous fistula with perimedullary venous drainage. AJNR Am J Neuroradiol. 1995; 16:2044-8.

7. Pirouzmand F, Wallace MC, Willinsky R. Spinal epidural arteriovenous fistula with intramedullary reflux. Case report. J Neurosurg. 1997; 87:633-5. 